\title{
Long-QT syndrome-associated caveolin-3 mutations differentially regulate the hyperpolarization-activated cyclic nucleotide gated channel 4
}

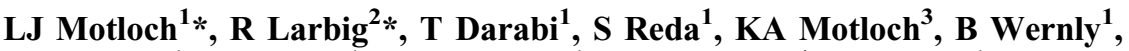 \\ M Lichtenauer ${ }^{1}$, T Gebing ${ }^{1}$, A Schwaiger ${ }^{1}$, N Zagidullin ${ }^{4}$, M Wolny ${ }^{1}$, UC Hoppe ${ }^{1}$ \\ ${ }^{1}$ Department of Internal Medicine II, Paracelsus Medical University, Salzburg, Austria \\ ${ }^{2}$ Division of Electrophysiology, Department of Cardiovascular Medicine, University Hospital Muenster, \\ Muenster, Germany \\ ${ }^{3}$ Research Program for Ophthalmology and Glaucoma Research, University Clinic of Ophthalmology and \\ Optometry, Paracelsus Medical University/SALK, Salzburg, Austria \\ ${ }^{4}$ Department of Internal Diseases, Bashkir State Medical University, Ufa, Russia
}

Received: August 17, 2016

Accepted: May 29, 2017

\begin{abstract}
Background: Caveolin-3 (cav-3) mutations are linked to the long-QT syndrome (LQTS) causing distinct clinical symptoms. Hyperpolarization-activated cyclic nucleotide channel 4 (HCN4) underlies the pacemaker current $I_{\mathrm{f}}$. It associates with cav-3 and both form a macromolecular complex. Methods: To examine the effects of human LQTS-associated cav-3 mutations on HCN4-channel function, HEK293-cells were cotransfected with HCN4 and wild-type (WT) cav-3 or a LQTS-associated cav-3 mutant (T78M, A85T, S141R, or F97C). HCN4 currents were recorded using the whole-cell patch-clamp technique. Results: WT cav-3 significantly decreased HCN4 current density and shifted midpoint of activation into negative direction. HCN4 current properties were differentially modulated by LQTS-associated cav-3 mutations. When compared with WT cav-3, A85T, F97C, and T78M did not alter the specific effect of cav-3, but S141R significantly increased HCN4 current density. Compared with WT cav-3, no significant modifications of voltage dependence of steady-state activation curves were observed. However, while WT cav-3 alone had no significant effect on HCN4 current activation, all LQTS-associated cav-3 mutations significantly accelerated $\mathrm{HCN} 4$ activation kinetics. Conclusions: Our results indicate that $\mathrm{HCN} 4$ channel function is modulated by cav-3. LQTS-associated mutations of cav-3 differentially influence pacemaker current properties indicating a pathophysiological role in clinical manifestations.
\end{abstract}

Keywords: long-QT syndrome, caveolin-3, hyperpolarization-activated cyclic nucleotide gated channel 4, $I_{\mathrm{f}}$, whole-cell current

\section{Introduction}

The congenital long-QT syndrome (LQTS) is a potentially fatal disease, which is characterized by delayed cardiac repolarization, prolonged QT interval, and ventricular tachyarrhythmias leading to sudden cardiac death. It is caused by mutations in several genes encoding protein subunits of cardiac ion channels (28).

\footnotetext{
*These authors contributed equally to this work.

Corresponding author: Lukas Jaroslaw Motloch, $\mathrm{MD}, \mathrm{PhD}$

Department of Internal Medicine II, Paracelsus Medical University

Müllner Hauptstrasse 48, Salzburg 5020, Austria

Phone: +43 6624482 58807; Fax: +43 6624482 4111; E-mail: lukas.motloch@pmu.ac.at
} 
Hyperpolarization-activated cyclic nucleotide channels $(\mathrm{HCN})$ underlie the cardiac pacemaker current $I_{\mathrm{f}}$ and play a central role in cardiac pacemaker activity (8). The HCN family consists of four mammalian isoforms (HCN1-4) $(7,8,18)$. Among them, HCN4 is known to be the dominant isoform of the sinus node region, which is responsible for spontaneous cardiac pacemaker activity (26).

Lipid rafts are microdomains of the plasma membranes, which consist of cholesterol and sphingolipids (12). They are responsible for the coordination of multiple cellular processes including second-messenger signaling and modulation of biophysical properties of ion channels. Caveolae are the lipid rafts composed of caveolin. By recruiting lipids and proteins that participate in intracellular trafficking, signal transduction as well as endo- and exocytosis, these scaffolding proteins are essential for the formation of caveolae complexes (2).

Cardiac sinus node cells contain a high density of caveolae (20). Furthermore, caveolae are known to modulate sympathetic response to the heart by colocalization of cellular key signaling molecules, such as G proteins, adenyl cyclase, and protein kinase A (25). A total of 3 members of the caveolin family are known to be essential for caveolae formation: caveolins 1, 2, and 3 (cav-1-3) (13). In cardiac tissue, caveolae formation depends on cav-3 (9), which colocalizes with different cardiac ion channels $(3,19,30)$. HCN4 is localized in calveolae and was shown to interact with cav-3 proteins $(4,5)$. Furthermore, calveolae disruption was proven to alter the gating function of HCN4 channels by shifting the voltage dependence of activation into positive direction (4).

Several mutations in cav-3 have been identified to associate with the congenital LQTS, namely, T78M, A85T, F97C, and S141R. In heterologous expression, these cav-3 mutations increased the late sodium current. Each mutation leads to specific clinical symptoms, such as sinus bradycardia and non-exertional syncope (T78M), cardiac arrest in sleep (A85T), shortness of breath and chest pain (F97C), or non-exertional syncope (S141R) (29).

In this study, we investigated the effects of human wild-type (WT) cav-3 and LQTSassociated cav-3 mutations on HCN4 ion channel function. We hypothesized cav-3 to influence HCN4 current characteristics. Furthermore, if cav-3 mutations distinctively impair electrophysiological HCN4 current properties, mutation-specific clinical manifestations might be explained. Therefore, to investigate the effects of cav-3 or LQTS-associated cav-3 mutations on HCN4 whole-cell current properties, we used heterologous expression of cav-3 or LQTS-associated cav-3 mutations cotransfected with HCN4 in the HEK293 cell system that naturally lacks cav-3 (25).

\section{Materials and Methods}

\section{Plasmid construction}

The expression plasmid pAdCGI-HCN4 (encoding the full-length sequences of HCN4 genes, respectively) as well as pAdCGI have previously been described $(7,8,15)$. The coding sequence of red fluorescent protein (RFP; pDsRed, Clontech, Saint-Germain-en-Laye, France) was cloned into pAdCGI in place of the enhanced green fluorescent protein (EGFP) sequence to generate pAdCRI. The full-length coding sequence of human cav-3 was amplified by polymerase chain reaction from human cardiac m-RNA, control sequenced, and cloned into the multiple cloning site of pAdCRI to generate pAdCRI-CAV-3. Cav-3 mutations (T78M, A85T, F97C, and S141R) were generated (11) using a QuickChange II XL Site-Directed Mutagenesis Kit (Stragene, The Netherlands) as directed by the manufacturer (11). 
Transient transfections

Twenty-four hours before transfection, HEK293 cells (American Type Culture Collection, Manassas, VA, USA) were seeded at a density of $2.0 \times 10^{5}$ cells $/ 35-\mathrm{mm}$ dish. The cells were cotransfected with $0.25 \mu \mathrm{g} /$ well plasmid DNA of HCN4 channels and $0.5 \mu \mathrm{g} /$ well plasmid DNA of cav-3, LQTS-associated cav-3 mutation, or an empty plasmid, as indicated, using Lipofectamine Plus (Life Technologies, Gaithersburg, MD, USA), as directed by the manufacturer. After $4 \mathrm{~h}$, the transfection medium was replaced by normal growth medium. The electrophysiological experiments were performed $48 \mathrm{~h}$ after the transfection.

\section{Patch-clamp recordings in whole-cell configuration}

The experiments were carried out using standard microelectrode whole-cell patch-clamp technique at room temperature $\left(21-23^{\circ} \mathrm{C}\right)$. The voltage error was compensated and currents were recorded and digitized with an Axopatch 200B amplifier and Digidata 1322 interface (Molecular Devices, Sunnyvale, CA, USA) while being sampled at $10 \mathrm{kHz}$ and filtered at $2 \mathrm{kHz}(7,8,14)$. The recording bath solution contained (in mmol/l) $135 \mathrm{NaCl}, 5 \mathrm{KCl}$, $2 \mathrm{CaCl}_{2}, 1 \mathrm{MgCl}_{2}, 10$ Glucose, and $10 \mathrm{HEPES} ; \mathrm{pH}$ was adjusted to 7.4 with $\mathrm{NaOH}$. The pipette solution contained (in mmol/l) $130 \mathrm{~K}$-glutamate, $5 \mathrm{KCl}, 5 \mathrm{NaCl}, 1 \mathrm{MgCl}_{2}, 10 \mathrm{HEPES}$, and $5 \mathrm{Mg}$-ATP; $\mathrm{pH}$ was adjusted to 7.3 with $\mathrm{KOH}$. Borosilicate microelectrodes had tip resistances of 2-4 M $\Omega$ when filled with pipette solution.

Only traces with a good signal quality were analyzed in this study. HCN4 current size was measured as the difference between the instantaneous current at the beginning of hyperpolarizing step ranging from -70 to $-160 \mathrm{mV}$ (or maximal $-170 \mathrm{mV}$ ) in $10-\mathrm{mV}$ decrements for $4.45-5 \mathrm{~s}$, and the steady-state current at the end of hyperpolarization, as previously described $(7,8,14)$. Fast-current inactivation was achieved by a depolarization pulse to $20 \mathrm{mV}$. Since most cells died when measurements at $-170 \mathrm{mV}$ were obtained, only traces recorded at hyperpolarizing steps ranging from -70 to $-160 \mathrm{mV}$ were analyzed for this study. The current density was calculated by the ratio of the measured current size relative to the cell capacitance ( $\mathrm{pA} / \mathrm{pF}$; mean cell capacitance: $46.37 \pm 3.55 \mathrm{pF}$ ). For the calculation of voltage dependence of steady-state activation curves, the specific current conductances $(g)$ were normalized to the maximal current conductance $\left(g_{\max }\right)$ to determine $g / g_{\max }$. Boltzmann distributions were fitted to these normalized values as follows: $g / g_{\max }=1 /\left\{1+\exp \left[\left(V_{1 / 2}-V_{\mathrm{m}}\right) / S\right]\right\}$, where $V_{1 / 2}$ is the voltage at half-maximal activation, $V_{\mathrm{m}}$ is the membrane voltage, and $\mathrm{S}$ is a slope factor at $V_{\mathrm{m}}=V_{1 / 2}$. To describe time constants for $I_{\mathrm{f}}$ activation ( $\tau$ values), current traces were fitted offline to determine $\tau$ values for test potentials of -110 to $-160 \mathrm{mV}$ using Prism 5 (Graphpad, San Diego, CA, USA) as previously described $(7,10)$. A xenon arc lamp was used to view EGFP at 488-nm excitation/530-nm emission and RFP at 557-nm excitation/579-nm emission. We observed no run-down phenomena during our recordings.

\section{Statistical analysis}

Pooled data are presented as mean \pm standard error of the mean. Comparisons between groups were performed with one-way ANOVA followed by post-hoc Tukey's test. Probability values of $p<0.05$ were regarded significant.

\section{Results}

To evaluate the effect of cav-3 on HCN4 whole-cell currents, HEK293 cells were transiently transfected with HCN4 in the presence of an empty control plasmid (CP) (to exclude non-specific 
effects), WT cav-3, or one of the LQTS-associated cav-3 mutations (T78M, A85T, F97C, or $\mathrm{S} 141 \mathrm{R})$. The combination of cytosolic green and red fluorescences verified effective cotransfection with both the plasmid-encoding HCN4 and the cav-3 construct, respectively.

Investigation of the LQTS-associated cav-3 mutations revealed differential modulations of HCN4 currents, which are summarized in Table I.

\section{Effects of cav-3 on HCN4 whole-cell current}

Cav-3 significantly decreased whole-cell current densities (Table I, Figs 1A, B and 2A) and significantly shifted the activation curve of $\mathrm{I}_{\mathrm{HCN} 4}$ to more negative values compared with

Table I. Whole-cell current parameters of HCN4 + CP, HCN4 + WT cav-3, HCN4 + cav-3 T78M, HCN4 + cav-3 A85T, HCN4 + cav-3 F97C, and HCN4 + cav-3 S141R

\begin{tabular}{|l|c|c|c|c|}
\hline HCN4+ & MCD $(\mathbf{p A} / \mathbf{p F})$ & $\tau(\mathbf{m s})$ & $V_{1 / 2 \max }(\mathbf{m V})$ & $n$ \\
\hline WT cav-3 & $-6.30 \pm 1.50$ & $2,948 \pm 354$ & $-139.1 \pm 4.0$ & 11 \\
\hline CP & $-22.02 \pm 2.66^{*}$ & $2,334 \pm 382$ & $-118.8 \pm 5.0^{*}$ & 13 \\
\hline cav-3 T78M & $-5.89 \pm 0.98$ & $1,073 \pm 271^{*}$ & $-129.0 \pm 1.9$ & 13 \\
\hline cav-3 A85T & $-13.70 \pm 2.43$ & $1,056 \pm 255^{*}$ & $-130.1 \pm 3.0$ & 9 \\
\hline cav-3 F97C & $-13.23 \pm 2.50$ & $1,077 \pm 434^{*}$ & $-138.3 \pm 2.8$ & 7 \\
\hline cav-3 S141R & $-34.49 \pm 6.35^{*}$ & $1,150 \pm 60^{*}$ & $-129.5 \pm 2.9$ & 7 \\
\hline
\end{tabular}

MCD: mean current density at $-130 \mathrm{mV} ; \tau$ : time constant of activation at $-130 \mathrm{mV} ; V_{1 / 2 \max }$ : voltage of half maximal activation.

$* p<0.05$ vs. HCN4 + WT cav-3

(A)

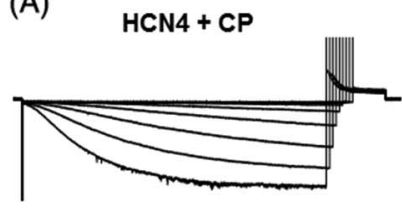

(D)

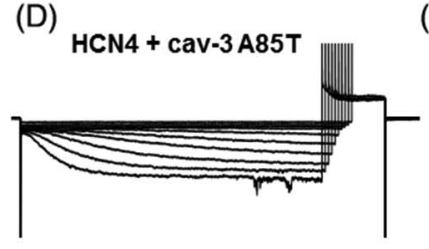

(B)

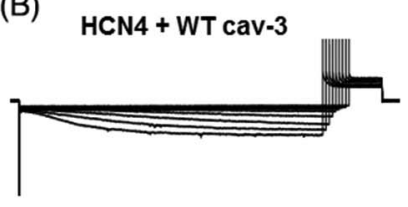

E)

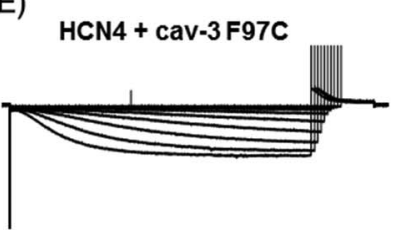

(C)

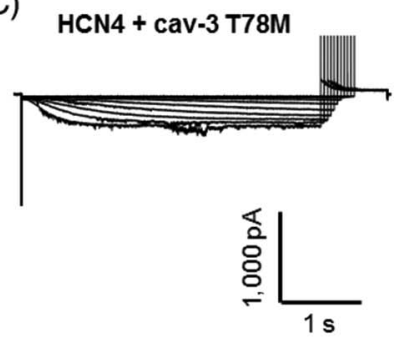

(F)

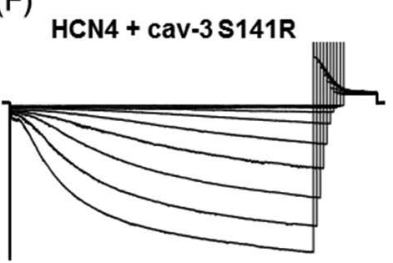

Fig. 1. Representative HCN4 whole-cell current recordings. $\mathrm{HCN} 4$ whole-cell current in the presence of (A) a $\mathrm{CP}$, (B) WT cav-3, (C) cav-3 T78M, (D) cav-3 A85T, (E) cav-3 F97C, or (F) cav-3 S141R. From a holding potential of $-40 \mathrm{mV}$, a hyperpolarization protocol by steps ranging from -70 to $-160 \mathrm{mV}$ in $10-\mathrm{mV}$ decrements followed by a depolarization step to $20 \mathrm{mV}$ is presented 


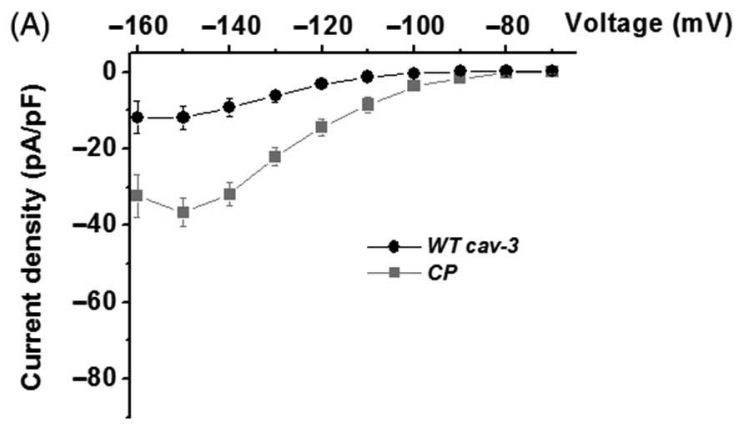

(B)

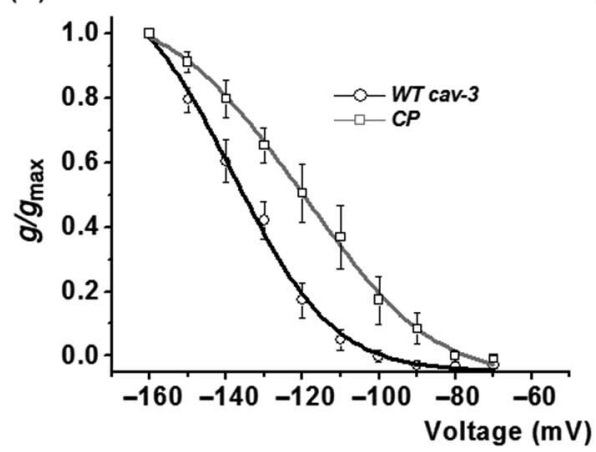

(C)

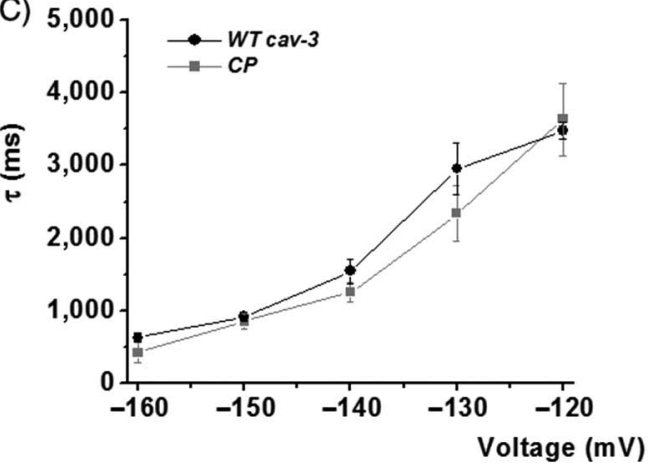

Fig. 2. Effects of WT cav-3 on HCN4 whole-cell current properties. (A) Mean HCN4 current density measured as the difference between the instantaneous current and steady-state current at the end of the hyperpolarization in the presence of WT cav-3 (black) or a CP (gray). (B) Voltage dependence of steady-state activation curves of HCN4 currents. The voltage dependence of HCN4 calculated by Boltzmann fits of normalized conductances $\left(\mathrm{g} / g_{\max }\right)$ was recorded in the presence of WT cav-3 (black) or a CP (gray). (C) Time constant $(\tau)$ values of current activation plotted as a function of test potentials in the presence of WT cav-3 (black) or a CP (gray)

transfection with CP (Table I, Fig. 2B). WT cav-3 had no significant impact on $\tau$ values of $\mathrm{I}_{\mathrm{HCN} 4}$ (Table I, Fig. 2C).

\section{Effects of cav-3 LQTS-associated mutations on HCN4 whole-cell current}

Compared with WT cav-3, the expression of cav-3 T78M, A85T, F97C, and S141R mutations had no significant impact on voltage dependence of steady-state activation curves of $\mathrm{I}_{\mathrm{HCN} 4}$ (Table I, Fig. 3B). Furthermore, T78M, A85T, and F97C did not significantly affect the whole-cell current densities (Table I, Figs 1C-E and 3A). However, when matched to WT cav-3, $\mathrm{I}_{\mathrm{HCN} 4}$ modulated by $\mathrm{S} 141 \mathrm{R}$ presented significantly increased whole-cell current densities (Table I, Figs $1 \mathrm{~F}$ and $3 \mathrm{~A}$ ). Of note, all four cav-3 LQTS-associated mutations (T78M, A85T, F97C, and S141R) significantly decreased $\tau$ values of $\mathrm{I}_{\mathrm{HCN} 4}$ indicating a faster activation kinetic of $\mathrm{I}_{\mathrm{HCN} 4}$ (Table I, Fig. 3C).

\section{Discussion}

Our results confirmed an interaction of HCN4 and cav-3. Importantly, compared with WT cav-3, LQTS-associated cav-3 mutations were shown to differentially influence pacemaker current properties (Table I). 

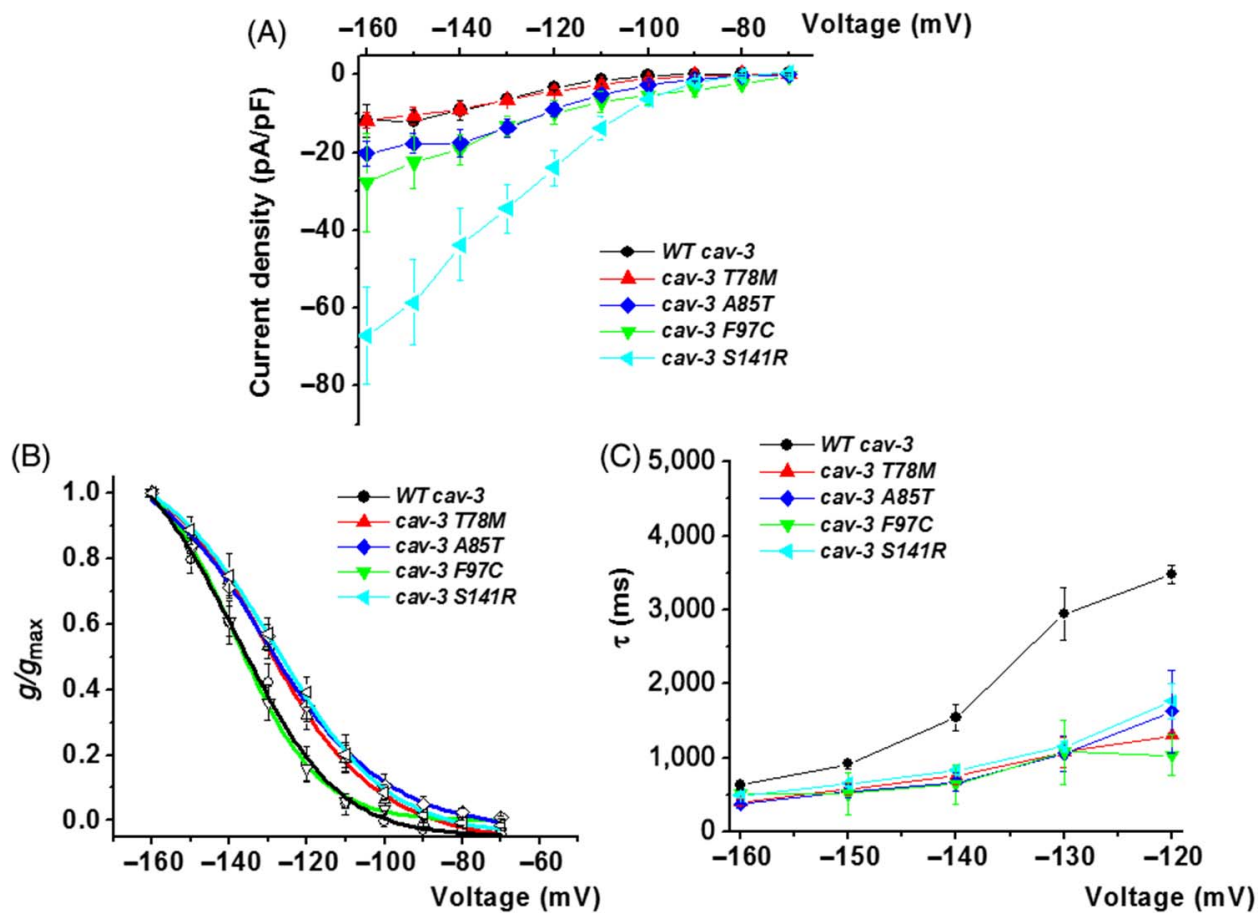

Fig. 3. Effects of WT cav-3 and LQTS-associated cav-3 mutations on HCN4 whole-cell current properties. (A) Mean HCN4 current density measured as the difference between the instantaneous current and steady-state current at the end of the hyperpolarization in the presence of WT cav-3, cav-3 T78M, cav-3 A85T, cav-3 F97C,

or cav-3 S141R. (B) Voltage dependence of steady-state activation curves of HCN4 currents. The voltage dependence of HCN4 calculated by Boltzmann fits of normalized conductances $\left(\mathrm{g} / \mathrm{g}_{\max }\right)$ was recorded in the presence of WT cav-3, cav-3 T78M, cav-3 A85T, cav-3 F97C, or cav-3 S141R. (C) Time constant $(\tau)$ values of current activation plotted as a function of test potentials in the presence of WT cav-3, cav-3 T78M, cav-3 A85T, cav-3 F97C, or cav-3 S141R

In this study, the expression of HCN4 and cav-3 in the HEK293 cell system, which naturally lacks cav-3 (25), demonstrated a direct modulation of $\mathrm{I}_{\mathrm{HCN} 4}$ by cav-3. This resulted in a significant negative shift in the $\mathrm{I}_{\mathrm{HCN} 4}$ activation curve and a significant decrease in $\mathrm{I}_{\mathrm{HCN} 4}$ whole-cell current density. Our observations are supported by previous findings. Importantly, consistent with our data, disruption of caveolae in rabbit sinoatrial myocytes as well as human embryonic stem cells resulted in a positive shift in the activation curve of $I_{\mathrm{f}}(4,6)$. Furthermore, cav-3 depletion was followed by an acceleration of spontaneous activity in cardiomyocytes including the sinoatrial node cells $(4,9)$. Therefore, one might speculate that cav-3 negatively regulates $\mathrm{I}_{\mathrm{HCN} 4}$ and probably $I_{\mathrm{f}}$ activity also.

$\mathrm{HCN}$ channels are responsible for the formation of the pacemaker current $I_{\mathrm{f}}(8,18)$. However, the expression of HCN isoforms in different systems gives rise to an inward current with similar but not identical characteristics compared with the native pacemaker current $(7,8)$. One of the reasons might be a modulation by $\beta$ subunits in vivo. Indeed, previous studies demonstrated the modulation of HCN channels by several $\beta$ subunits, which, interestingly similar to cav-3, are known to be associated with LQTS $(1,7,16,21)$. 
Therefore, our data might suggest that, among others, cav-3 interacts with HCN4 to modulate the channel's activity and thus support the rise of the native pacemaker current in vivo. Of note, HCN4 channels are the dominant isoform of the sinus node region (26), which is primarily responsible for the spontaneous cardiac pacemaker activity. Indeed, cav-3 and HCN4 were shown to associate in the pacemaker and the conduction system of different species including human $(4,6,22)$. Therefore, one might speculate that under physiological conditions, cav-3 suppresses $\mathrm{I}_{\mathrm{HCN} 4}$ activity leading to slower heart rates. These speculations are further supported by studies of spontaneous activity in cav-3-depleted cardiomyocytes including the sinoatrial node cells, as previously mentioned $(4,9)$.

Furthermore, to explore potential generation of symptoms in patients with cav3-associated LQTS mutations, in this trial, their impact on $\mathrm{I}_{\mathrm{HCN} 4}$ function was investigated. Cav-3 mutations, such as T78M, A85T, F97C, and S141R, differentially affected gating parameters of $\mathrm{I}_{\mathrm{HCN} 4}$. However, compared with WT cav-3, A85T, F97C, and T78M did not alter current densities, but S141R significantly increased the current density of $\mathrm{I}_{\mathrm{HCN} 4}$. Importantly, all cav-3 mutations significantly accelerated activation kinetics of HCN4. Therefore, our results might indicate that these mutations undermine WT cav-3-mediated effects by probably increasing $\mathrm{I}_{\mathrm{HCN} 4}$ activity using a mutationspecific mechanism.

These observations could not explain sinus bradycardia as observed in carriers of T78M cav-3 mutation (29). However, one might speculate that by establishing a more active $\mathrm{I}_{\mathrm{HCN} 4}$, LQTS-associated cav-3 mutations increase $I_{\mathrm{f}}$ function and therefore accelerate the rate of spontaneous diastolic depolarization in the sinus node region. This might induce an increase in heart rate leading to sinus tachycardia. Sinus tachycardia is known to provoke symptoms like angina and shortness of breath, which indeed are associated with mutations of cav-3 in F97C (29). Furthermore, in LQTS accelerations of heart rate are described to provoke ventricular arrhythmias (24). Therefore, these events might contribute to the generation of ventricular tachycardia in LQTS patients leading to non-exertional syncope and cardiac arrest in sleep as well as angina, and shortness of breath (27), which are observed in patients with S141R, T78M, A85T, and F97C cav-3 mutations, respectively (29). In addition, one might speculate a further mechanism of how an increase in HCN4 current activity could contribute to the generation of spontaneous ventricular tachycardias in LQTS patients. Besides atrial, also ventricular myocytes express a small amount of HCN channels amog them HCN4 (26). Of note, increased $\mathrm{I}_{\mathrm{HCN} 4}$ activity in the ventricle was shown to provoke ventricular automaticity resulting in ventricular arrhythmias like ventricular tachycardia $(17,23)$. However, these suggestions were not the matter of investigation in this study and required to be addressed in further trials.

In summary, we provide an evidence for a direct interaction of WT cav-3 and HCN4 indicating that WT cav-3 negatively modulates $\mathrm{I}_{\mathrm{HCN} 4}$. This observation might be important for the formation of physiological pacemaker currents in vivo. Furthermore, we demonstrated LQTS-associated cav-3 mutations to differentially modulate HCN4 channel function indicating a pathophysiological role in clinical manifestations.

\section{Acknowledgement}

None. 


\section{REFERENCES}

1. Abbott GW, Sesti F, Splawski I, Buck ME, Lehmann MH, Timothy KW, Keating MT, Goldstein SA: MiRP1 forms IKr potassium channels with HERG and is associated with cardiac arrhythmia. Cell 97, 175-187 (1999)

2. Anderson RG: The caveolae membrane system. Annu. Rev. Biochem. 67, 199-225 (1998)

3. Balijepalli RC, Foell JD, Hall DD, Hell JW, Kamp TJ: Localization of cardiac L-type $\mathrm{Ca}^{2+}$ channels to a caveolar macromolecular signaling complex is required for $\beta_{2}$-adrenergic regulation. Proc. Natl. Acad. Sci. U S A $103,7500-7505$ (2006)

4. Barbuti A, Gravante B, Riolfo M, Milanesi R, Terragni B, DiFrancesco D: Localization of pacemaker channels in lipid rafts regulates channel kinetics. Circ. Res. 94, 1325-1331 (2004)

5. Barbuti A, Terragni B, Brioschi C, DiFrancesco D: Localization of f-channels to caveolae mediates specific $\beta_{2}$-adrenergic receptor modulation of rate in sinoatrial myocytes. J. Mol. Cell. Cardiol. 42, 71-78 (2007)

6. Bosman A, Sartiani L, Spinelli V, Del Lungo M, Stillitano F, Nosi D, Mugelli A, Cerbai E, Jaconi M: Molecular and functional evidence of HCN4 and caveolin-3 interaction during cardiomyocyte differentiation from human embryonic stem cells. Stem Cells Dev. 22, 1717-1727 (2013)

7. Brandt MC, Endres-Becker J, Zagidullin N, Motloch LJ, Er F, Rottlaender D, Michels G, Herzig S, Hoppe UC: Effects of KCNE2 on HCN isoforms: distinct modulation of membrane expression and single channel properties. Am. J. Physiol. Heart Circ. Physiol. 297, H355-H363 (2009)

8. Er F, Larbig R, Ludwig A, Biel M, Hofmann F, Beuckelmann DJ, Hoppe UC: Dominant-negative suppression of HCN channels markedly reduces the native pacemaker current $I_{\mathrm{f}}$ and undermines spontaneous beating of neonatal cardiomyocytes. Circulation 107, 485-489 (2003)

9. Galbiati F, Engelman JA, Volonte D, Zhang XL, Minetti C, Li M, Hou H Jr, Kneitz B, Edelmann W, Lisanti MP: Caveolin-3 null mice show a loss of caveolae, changes in the microdomain distribution of the dystrophinglycoprotein complex, and t-tubule abnormalities. J. Biol. Chem. 276, 21425-21433 (2001)

10. Gao LL, McMullan S, Djouhri L, Acosta C, Harper AA, Lawson SN: Expression and properties of hyperpolarization-activated current in rat dorsal root ganglion neurons with known sensory function. J. Physiol. 590, 4691-4705 (2012)

11. Greener A, Callahan M, Jerpseth B: An efficient random mutagenesis technique using an E. coli mutator strain. Mol. Biotechnol. 7, 189-195 (1997)

12. Harder T, Simons K: Caveolae, DIGs, and the dynamics of sphingolipid-cholesterol microdomains. Curr. Opin. Cell Biol. 9, 534-542 (1997)

13. Hnasko R, Lisanti MP: The biology of caveolae: lessons from caveolin knockout mice and implications for human disease. Mol. Interv. 3, 445-464 (2003)

14. Hoppe UC, Jansen E, Sudkamp M, Beuckelmann DJ: Hyperpolarization-activated inward current in ventricular myocytes from normal and failing human hearts. Circulation 97, 55-65 (1998)

15. Hoppe UC, Marban E, Johns DC: Distinct gene-specific mechanisms of arrhythmia revealed by cardiac gene transfer of two long QT disease genes, HERG and KCNE1. Proc. Natl. Acad. Sci. U S A 98, 5335-5340 (2001)

16. Isbrandt D, Friederich P, Solth A, Haverkamp W, Ebneth A, Borggrefe M, Funke H, Sauter K, Breithardt G, Pongs O, Schulze-Bahr E: Identification and functional characterization of a novel KCNE2 (MiRP1) mutation that alters HERG channel kinetics. J. Mol. Med. 80, 524-532 (2002)

17. Kuwabara Y, Kuwahara K, Takano M, Kinoshita H, Arai Y, Yasuno S, Nakagawa Y, Igata S, Usami S, Minami T, Yamada Y, Nakao K, Yamada C, Shibata J, Nishikimi T, Ueshima K, Nakao K: Increased expression of HCN channels in the ventricular myocardium contributes to enhanced arrhythmicity in mouse failing hearts. J. Am. Heart Assoc. 2, e000150 (2013)

18. Ludwig A, Zong X, Stieber J, Hullin R, Hofmann F, Biel M: Two pacemaker channels from human heart with profoundly different activation kinetics. EMBO J. 18, 2323-2329 (1999)

19. Martens JR, Sakamoto N, Sullivan SA, Grobaski TD, Tamkun MM: Isoform-specific localization of voltage-gated $\mathrm{K}^{+}$channels to distinct lipid raft populations. Targeting of Kv1.5 to caveolae. J. Biol. Chem. 276, 8409-8414 (2001)

20. Masson-Pevet M, Gros D, Besselsen E: The caveolae in rabbit sinus node and atrium. Cell Tissue Res. 208, 183-196 (1980)

21. Michels G, Er F, Khan IF, Endres-Becker J, Brandt MC, Gassanov N, Johns DC, Hoppe UC: $\mathrm{K}^{+}$channel regulator KCR1 suppresses heart rhythm by modulating the pacemaker current $I_{\mathrm{f}}$. PLoS One 3, e1511 (2008)

22. Mitrofanova LB, Gorshkov AN, Lebedev DS, Mikhaylov EN: Evidence of specialized tissue in human interatrial septum: histological, immunohistochemical and ultrastructural findings. PLoS One 9, e113343 (2014) 
23. Plotnikov AN, Bucchi A, Shlapakova I, Danilo P Jr, Brink PR, Robinson RB, Cohen IS, Rosen MR: HCN212channel biological pacemakers manifesting ventricular tachyarrhythmias are responsive to treatment with $I_{\mathrm{f}}$ blockade. Heart Rhythm 5, 282-288 (2008)

24. Roden DM, Lazzara R, Rosen M, Schwartz PJ, Towbin J, Vincent GM: Multiple mechanisms in the long-QT syndrome. Current knowledge, gaps, and future directions. The SADS Foundation Task Force on LQTS. Circulation 94, 1996-2012 (1996)

25. Rybin VO, Xu X, Lisanti MP, Steinberg SF: Differential targeting of $\beta$-adrenergic receptor subtypes and adenylyl cyclase to cardiomyocyte caveolae. A mechanism to functionally regulate the cAMP signaling pathway. J. Biol. Chem. 275, 41447-41457 (2000)

26. Shi W, Wymore R, Yu H, Wu J, Wymore RT, Pan Z, Robinson RB, Dixon JE, McKinnon D, Cohen IS: Distribution and prevalence of hyperpolarization-activated cation channel $(\mathrm{HCN})$ mRNA expression in cardiac tissues. Circ. Res. 85, e1-e6 (1999)

27. Task Force for the Diagnosis and Management of Syncope, European Society of Cardiology (ESC), European Heart Rhythm Association (EHRA), Heart Failure Association (HFA), Heart Rhythm Society (HRS), Moya A, Sutton R, Ammirati F, Blanc JJ, Brignole M, Dahm JB, Deharo JC, Gajek J, Gjesdal K, Krahn A, Massin M, Pepi M, Pezawas T, Ruiz Granell R, Sarasin F, Ungar A, van Dijk JG, Walma EP, Wieling W: Guidelines for the diagnosis and management of syncope (version 2009). Eur. Heart J. 30, 2631-2671 (2009)

28. Towbin JA, Vatta M: Molecular biology and the prolonged QT syndromes. Am. J. Med. 110, 385-398 (2001)

29. Vatta M, Ackerman MJ, Ye B, Makielski JC, Ughanze EE, Taylor EW, Tester DJ, Balijepalli RC, Foell JD, Li Z, Kamp TJ, Towbin JA: Mutant caveolin-3 induces persistent late sodium current and is associated with long-QT syndrome. Circulation 114, 2104-2112 (2006)

30. Yarbrough TL, Lu T, Lee HC, Shibata EF: Localization of cardiac sodium channels in caveolin-rich membrane domains: regulation of sodium current amplitude. Circ. Res. 90, 443-449 (2002) 